\title{
Chemical modification of the polymer of intrinsic microporosity PIM-1 for enhanced hydrogen storage
}

\author{
Mi $\operatorname{Tian}^{1,5}$ (D) Sébastien Rochat ${ }^{2,3} \cdot$ Hamish Fawcett ${ }^{1} \cdot$ Andrew D. Burrows $^{2} \cdot$ Christopher R. Bowen $^{4}$. \\ Timothy J. Mays ${ }^{1}$ iD
}

Received: 17 September 2019 / Revised: 28 April 2020 / Accepted: 8 May 2020 / Published online: 1 June 2020

(c) The Author(s) 2020

\begin{abstract}
A detailed investigation has been carried out of the pre-polymerisation modification of the polymer of intrinsic microporosity PIM-1 by the addition of two methyl (Me) groups to its spirobisindane unit to create a new chemically modified PIM-1 analogue, termed MePIM. Our work explores the effects of this modification on the porosity of PIM-1 and hence on its gas sorption properties. MePIM was successfully synthesised using either low (338 K) or high (423 K) temperature syntheses. It was observed that introduction of methyl groups to the spirobisindane part of PIM-1 generates additional microporous spaces, which significantly increases both surface area and hydrogen storage capacity. The BET surface area $\left(\mathrm{N}_{2}\right.$ at $\left.77 \mathrm{~K}\right)$ was increased by $\sim 12.5 \%$, resulting in a $\sim 25 \%$ increase of hydrogen adsorption after modification. MePIM also maintains the advantages of good processability and thermal stability. This work provides new insights on a facile polymer modification that enables enhanced gas sorption properties.
\end{abstract}

Keywords Hydrogen storage · Porous polymers · Polymer of intrinsic microporosity · PIM-1 · Modified PIM-1

\section{Introduction}

The preparation of new porous polymer membranes is receiving increasing attention by both academia and industry as a result of their potential for a number of important

Electronic supplementary material The online version of this article (https://doi.org/10.1007/s10450-020-00239-y) contains supplementary material, which is available to authorized users.

Timothy J. Mays

t.j.mays@bath.ac.uk

1 Department of Chemical Engineering, University of Bath, Claverton Down, Bath BA2 7AY, UK

2 Department of Chemistry, University of Bath, Claverton Down, Bath BA2 7AY, UK

3 Department of Engineering Mathematics, School of Chemistry, Bristol Composites Institute, University of Bristol, Bristol BS8 1UB, UK

4 Department of Mechanical Engineering, University of Bath, Claverton Down, Bath BA2 7AY, UK

5 Present Address: College of Engineering, Mathematics and Physical Sciences, University of Exeter, Harrison Building, Streatham Campus, N Park Rd, Exeter EX4 4QF, UK applications. These include hydrogen storage (Rochat et al. 2017), hydrogen production and separation (Xu et al. 2017), $\mathrm{CO}_{2}$ separation and storage (Budd et al. 2005), and waste gas treatment (Kim et al. 2016). Solvent-processable adsorbents are advantageous over powders in terms of safety, handling and practical ease of large-scale manufacturing.

Polymers of intrinsic microporosity (PIMs) are a widely investigated class of polymers with backbones composed of rigid ladder-like sequences with sites of contortion, which prohibits efficient packing in the solid state and creates a large free volume. Consequently, PIMs exhibit relatively high surface areas within the range of 560-1760 $\mathrm{m}^{2} \mathrm{~g}^{-1}$ and micropores of size below $2 \mathrm{~nm}$ (Rochat et al. 2017; Budd et al. 2005,2004; Tian et al. 2019), as defined by International Union of Pure and Applied Chemistry (IUPAC) (Matthias et al. 2015). The archetypal PIM, referred to as PIM-1, is soluble in organic solvents such as chloroform and tetrahydrofuran, and can be processed into membranes and even into fibre form via electrospinning (Satilmis et al. 2019). Despite its attractive intrinsic properties and processability, PIM-1 still exhibits relatively low hydrogen storage properties ( $<2$ wt\% Rochat et al. 2017) due to limiting surface area and pore volume, the enhancement of which remains a challenging task. Earlier attempts to improve the surface 
area and gas storage properties of PIM-1 involved mixing or filling it with a very high surface area powder, such as metalorganic frameworks (MOFs) or activated carbon, to create a composite membrane. Some examples of this include our previous work in mixing PIM-1 and porous aromatic frameworks (PAF-1) (Rochat et al. 2017) and a commercial activated carbon (AX21) (Tian et al. 2019) to produce composite membranes with increased hydrogen uptake capacity while maintaining the processability of PIM- 1 . Khdhayyer et al. investigated PIM-1 and UiO-66 for a gas separation application, leading to a high gas permeability of the composite PIM-1 based membrane (Khdhayyer et al. 2017).

The intrinsic properties of PIM-1 may also be modified through cross-linking. For example, Song et al. reported that controllable thermal oxidative crosslinking of PIMs resulted in covalently crosslinked networks, showing remarkable gas selectivity (Song 2014). Thermal treatment under an inert atmosphere has been shown to lead to cross-linked products, resulting in dramatic changes in properties. However, the presence of cross-linking may damage microporosity and have a negative impact on the gas storage properties of PIM-1 (Song 2014).

The modification methods mentioned above have often either reduced the surface area of PIM-1, or have led to inferior mechanical properties. Another approach to modifying PIM-1 is by post-synthetic modification of the nitrile groups to $\mathrm{CO}_{2}$-philic functionalities. (Du et al. 2009). A variety of reactions can be carried out on the nitrile group, including hydrolysis. Swaidan et al. (Swaidan et al. 2014) found that $\mathrm{CO}_{2}$-philic amidoxime functionalization improved selectivity for $\mathrm{CO}_{2}$ over $\mathrm{CH}_{4}$ due to the enhanced interchain hydrogen bonding offered by the amidoxime moieties. More rigid groups, such as Tröger's base (Wang et al. 2014), have been introduced in the polymer backbone to achieve high selectivity for $\mathrm{CO}_{2}$ separation without compromising the surface area. The introduction of Tröger's base provides the double ladder PIM polymer a more rigid and contorted structure, resulting in an enhanced intrinsic microporosity (Wang et al. 2014). Halder et al. (Halder et al. 2018) modified PIM-1 by the addition of vinyl groups to the tetrahydroxy monomer before polymerisation steps of PIM-1 to obtain the vinyl homo- and copolymers. The addition of vinyl groups acted to stiffen the polymer backbone, which improved selectivity for gas separation. Prior examples of PIM-1 modifications have focussed on tailoring its selectivity for gas or liquid separations (Satilmis and Budd 2017). However, the hydrogen storage properties of the modified PIMs have yet to be studied.

In this work, we have investigated a structurally modified PIM-1 by the addition of two methyl groups to the spirobisindane monomer, to create MePIM at low (338 K) and high $(423 \mathrm{~K})$ temperature whilst maintaining the processability and thermal stability of PIM-1. Our work uses a similar synthetic approach reported by Halder et al. (Halder et al. 2018); however, Halder et al. investigated mainly synthesis and $\mathrm{CO}_{2}$ separation properties of the modified PIMs but showed little information on surface areas, porosity and gas adsorption properties. In this work, we studied the surface area and porosity of the modified PIMs in detail and provide the first investigation of their hydrogen storage properties.

\section{Experimental section}

\subsection{Materials}

All chemicals used in this work were sourced from SigmaAldrich, Fluorochem, VMR or Strem Chemicals, and used without further purification, unless indicated.

PIM-1 was synthesised following a published procedure (Budd et al. 2004). $6.0 \mathrm{~g}$ (29.4 mmol) of tetrafluoroterephthalonitrile (TFN, previously purified by recrystallization from hot acetone), $10.22 \mathrm{~g}(29.2 \mathrm{mmol})$ of 3,3,3',3'-tetramethyl$1,1^{\prime}$-spirobisindane-5,5',6,6'-tetraol and $33.2 \mathrm{~g}$ of anhydrous $\mathrm{K}_{2} \mathrm{CO}_{3}$ were stirred under an atmosphere of dry $\mathrm{N}_{2}$ at 338 $\mathrm{K}$ for 3 days in $100 \mathrm{ml}$ of dry dimethylformamide (DMF). On cooling, the suspension was separated by filtration and washed with water followed by acetone. After drying at 80 ${ }^{\circ} \mathrm{C}$ overnight in a vacuum oven, the solid was dissolved in $200 \mathrm{ml}$ of chloroform and precipitated into $1.8 \mathrm{~L}$ of methanol. The reprecipitation procedure was performed three times, and PIM-1 was finally collected as bright yellow granules.

Methylated spirobisindane monomer was synthesised using the method reported by Halder et al. (Halder et al. 2018). $20 \mathrm{~g}$ of 3-methylcatechol was suspended in $40 \mathrm{ml}$ AcOH followed by adding $45 \mathrm{ml} \mathrm{HBr}$ (48\% in water), which resulted in a clear solution. $26 \mathrm{ml}$ of acetone was then added dropwise over 15-30 min using a dropping funnel, resulting in the formation of a dark brown solution. The solution was further heated at $393 \mathrm{~K}$ for $12 \mathrm{~h}$ to complete the reaction. The monomer was precipitated into 21 water, then separated by filtration and washed with water followed by several washes with diethyl ether. The white powder formed was then dried under reduced pressure at room temperature.

MePIM65 and MePIM150 (65 and 150 correspond to reaction temperature 65 and $150{ }^{\circ} \mathrm{C}$ ) were synthesised using a method similar to the PIM-1 synthesis described above, apart from using the methylated spirobisindane monomer instead of 5,5',6,6'-tetrahydroxy-3,3,3',3'tetramethylspirobisindane. Methylated spirobisindane monomer $(5.38 \mathrm{~g}, 14.6 \mathrm{mmol})$, TFN $(3.0 \mathrm{~g}, 14.7 \mathrm{mmol})$ and anhydrous $\mathrm{K}_{2} \mathrm{CO}_{3}(16.6 \mathrm{~g})$ were stirred under dry $\mathrm{N}_{2}$ atmosphere in $100 \mathrm{ml}$ dry DMF at $338 \mathrm{~K}\left(65^{\circ} \mathrm{C}\right)$ for 3 days (to form MePIM65), or in dimethylacetamide (DMA) at $423 \mathrm{~K}$ (150 
${ }^{\circ} \mathrm{C}$ ) for $45 \mathrm{~min}$ (to form MePIM150). After the reactions were complete, the bright yellow mixture that formed was precipitated into $300 \mathrm{ml}$ water and separated by filtration, washed with water and then acetone. The crude polymers were dissolved in $60 \mathrm{ml}$ chloroform and precipitated dropwise using a dropping funnel into $600 \mathrm{ml}$ of methanol. The product was then separated by filtration, and the retentate was dried at $338 \mathrm{~K}$ overnight in a vacuum oven. This reprecipitation process was repeated a total of three times, and the polymers were finally isolated as bright yellow granular solids.

\subsection{Membrane casting}

The MePIM membranes were cast using a previously described protocol designed for PIM-1 (Rochat et al. 2017; Tian et al. 2019). Briefly, the MePIM powders were dissolved in chloroform to form a $2 \mathrm{wt} \%$ mixture that was stirred for $24 \mathrm{~h}$ at room temperature. The solutions were then cast into a Teflon dish covered with a lid and left to evaporate in a desiccator, resulting in $400 \mathrm{mg}$ films. The formed membranes were then dried under reduced pressure at $353 \mathrm{~K}$ for $8 \mathrm{~h}$. Finally, the membranes were peeled away from the Petri dish for measurement and testing.

\subsection{Characterisation}

${ }^{1} \mathrm{H}$ NMR analyses were carried out in deuterated chloroform $\left(\mathrm{CDCl}_{3}\right)$ at room temperature with an Agilent ProPulse $500 \mathrm{MHz}$ spectrometer using residual solvent as an internal reference.

Gel permeation chromatography (GPC) was performed in tetrahydrofuran (THF) at $1 \mathrm{ml} / \mathrm{min}$ and $308 \mathrm{~K}$ with an Agilent Technologies 1260 Infinity instrument calibrated against polystyrene standards and equipped with refractive index, light scattering and viscosity detectors. Molecular weight distributions were calculated using the triple-detection mode of the Agilent GPC/SEC software. The ratio of average molecular weight $(\mathrm{Mw})$ to number average molar mass (Mn), Mw/Mn, gives the polydispersity index (PDI).

All samples were degassed at $353 \mathrm{~K}$ under a vacuum of $0.1 \mathrm{mPa}$ in order to remove moisture and adsorbed gases from the surface before the following measurements.

Thermogravimetric analysis (TGA) was performed in flowing air at $20 \mathrm{ml} / \mathrm{min}$ up to $1073 \mathrm{~K}$ at $10 \mathrm{~K} / \mathrm{min}$ using a Setaram Setsys Evolution 16 TGA instrument. The results were corrected for buoyancy by subtracting blank traces under the same conditions.

Scanning electron microscopy (SEM) analysis of the membranes to study their cross-section morphology was performed using a Field Emission-SEM (FESEM), JEOL JSM-6301F instrument. The samples were coated with a 10 nm chromium layer via sputtering, using a Quorum Q150TS. Cross-sections of membrane samples were prepared by freeze fracturing after immersion in liquid nitrogen. The fractured samples were mounted on stubs using two-side conductive carbon tape.

Surface areas were determined by BET analysis with $\mathrm{N}_{2}$ isotherms at $77 \mathrm{~K}$ using a Micrometritics 3Flex volumetric gas sorption analyser $\left(p / p_{0}\right.$ range used for the BET calculation: $0.01-0.15$ ). All samples were degassed in-situ at $333 \mathrm{~K}$ for 8 $\mathrm{h}$ before each measurement on the 3Flex. Total pore volumes were calculated from the nitrogen uptake at $p / p_{0}=0.97$, and pore size distributions were estimated using non-local density functional theory (NLDFT) provided with the MicroActive data analysis software version 3.0 using a slit-pore model for $\mathrm{N}_{2}$ at $77 \mathrm{~K}$. The $\mathrm{N}_{2}$ gas (oxygen free) with a purity of $99.998 \%$ and $\mathrm{He}$ gas with a purity of $99.996 \%$ were supplied by BOC Ltd. The free space was measured using $\mathrm{He}$ gas at $77 \mathrm{~K}$ and at room temperature for three times to determine the average free space. The average free space was used for sample volume correction using the 3Flex software built-in calculation. All the isotherms present in this paper have been corrected by the free space measurement.

$\mathrm{CO}_{2}$ adsorption up to $2 \mathrm{MPa}$ at $273 \mathrm{~K}$ was carried out on an Intelligent Gravimetric Analyzer (IGA-002) from Hiden Isochema, Warrington, UK. An ice bath was used for temperature control. The measured $\mathrm{CO}_{2}$ isotherms data were imported to the MicroActive data analysis software (Micromeritics) V3.0 for pore size distribution analysis using the slit-pore model for $\mathrm{CO}_{2}$ at $273 \mathrm{~K}$ (NLDFT). $\mathrm{CO}_{2}(99.8 \%)$ was purchased from BOC Ltd.

Low-pressure hydrogen adsorption measurements (up to $0.1 \mathrm{MPa}$ ) at $77 \mathrm{~K}$ were carried out using a 3Flex instrument from Micromeritics. The high-pressure hydrogen isotherms at $77 \mathrm{~K}$ (up to $12 \mathrm{MPa}$ ) were collected at equilibrium in a Hiden HTP-1 volumetric sorption analyser using a liquid nitrogen immersion Dewar for temperature control (Hiden Isochema, Warrington, UK). Prior to hydrogen uptake measurements, the samples $(\sim 150 \mathrm{mg})$ were degassed in situ at $353 \mathrm{~K}$ for $8 \mathrm{~h}$ under a vacuum of $0.1 \mathrm{mPa}$ in order to remove moisture and adsorbed gases from the surface. The hydrogen isotherms at $77 \mathrm{~K}$ are fully reproducible and reversible within $\pm 0.3 \%$ of measured amounts adsorbed. Nonlinear fitting and calculations were carried out using a Levenberg-Marquardt method via OriginPro 2018. Hydrogen gas used for all measurement is BIP PLUS grade high-purity $99.999996 \%$ hydrogen from Air Products. The isotherms were analysed and modelled using our previously reported methodology (Rochat et al. 2017; Tian et al. 2019) in order to estimate the total hydrogen adsorption capacity and the adsorbed $\mathrm{H}_{2}$ density, which is reported in the supporting information, along with details of the modelling (Figure S2 and Table S1). 


\section{Results and discussion}

The modified spirobisindane monomer was synthesised from 3-methyl catechol and acetone, as described in Scheme 1 and the experimental section.

The reaction route to MePIM was based on the high temperature PIM-1 synthesis, shown in Scheme 2. The yields of MePIM65 and MePIM150 were 94 and 99\%, respectively. The colour of MePIM65 was light yellow, compared to the dark yellow granular colour of MePIM150. In a control experiment, PIM-1 was also prepared as a comparison following a previously reported method (Rochat et al. 2017).

The molar masses as characterised by GPC are summarised in Table 1. The molar mass and the polydispersity index (PDI) of the synthesized PIM-1 is consistent with the reported studies (Budd et al. 2004; Du et al. 2010; PolakKraśna et al. 2017), and has a considerably higher average molar mass than the MePIMs. We attribute these differences to a probable lower solubility of MePIMs in the reaction medium compared to PIM-1. This explanation is supported by the fact that MePIM150 has a higher molar mass than
MePIM65, which can be attributed to the higher reaction temperature used for its synthesis $(423 \mathrm{~K} v$ s. $338 \mathrm{~K})$. Comparative ${ }^{1} \mathrm{H}$ NMR spectra of PIM-1 and MePIMs are shown in Fig. 1, suggesting successful synthesis and good purity of PIM-1 and both MePIMs, as chemical shifts appear consistent with published spectra of PIM-1, intensities are consistent with the proposed structures, and the broadened peaks are typical of polymer materials (Halder et al. 2018; Du et al. 2010; Polak-Kraśna et al. 2017). Thermogravimetric analysis, Fig. 2, shows MePIMs are thermally stable and decomposition of MePIM begins at $603 \mathrm{~K}\left(330{ }^{\circ} \mathrm{C}\right)$ in air, slightly lower than PIM-1 (begins at $673 \mathrm{~K}$ ).

The MePIMs maintain good solubility in organic solvents and have been successfully cast into homogeneous membranes with thicknesses of $200-500 \mu \mathrm{m}$ using our previously developed film casting method (Polak-Kraśna et al. 2017). However, it was observed during handling that the MePIM membranes were more brittle than the pure PIM-1 membranes.

Figure 3 shows SEM images of the cross section of PIM-1 and the MePIMs, suggesting they are cast as relatively uniform materials. At high magnification, the cross sections

Scheme 1 Synthesis of the methylated spirobisindane monomer

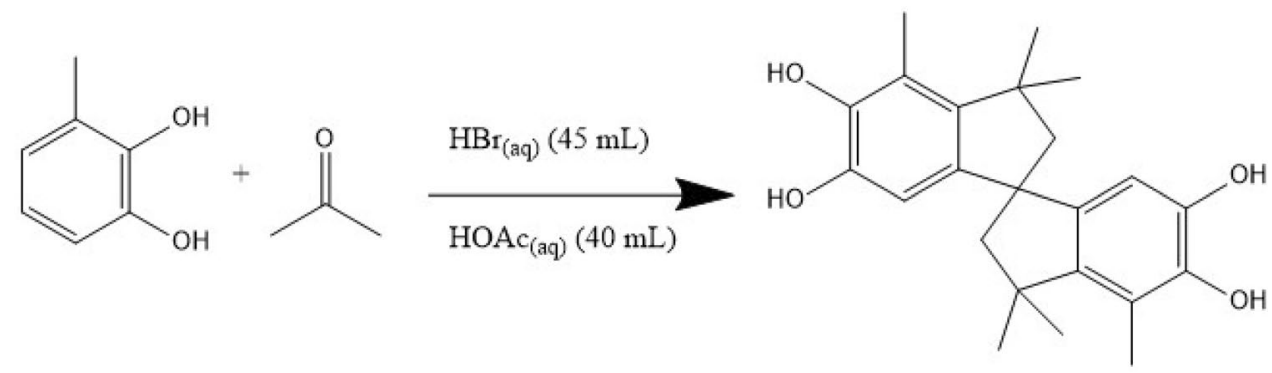

MePIM65

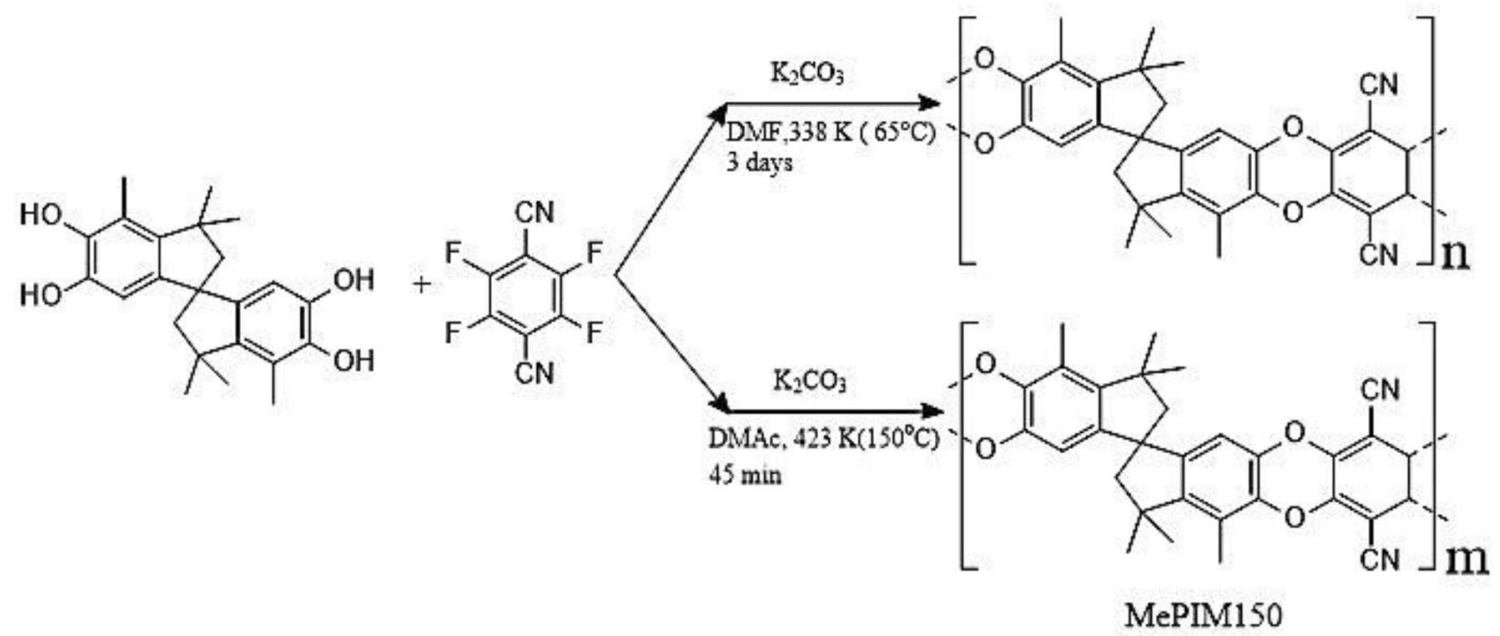

Scheme 2 Synthesis of MePIM65 and MePIM15 
Table 1 Molecular, surface and $\mathrm{H}_{2}$ uptake properties of the polymers investigated

\begin{tabular}{lllllllll}
\hline Polymer & $\begin{array}{l}\text { Reaction } \\
\text { temperature } \\
(\mathrm{K})\end{array}$ & $\left.\mathrm{M}_{\mathrm{w}}(\mathrm{g} \mathrm{mol})^{-1}\right)$ & $\mathrm{PDI}\left(\mathrm{M}_{\mathrm{n}} / \mathrm{M}_{\mathrm{w}}\right)$ & $\mathrm{S}_{\mathrm{BET}}\left(\mathrm{m}^{2} \mathrm{~g}^{-1}\right)^{\mathrm{a}}$ & $\mathrm{V}_{\text {total }}\left(\mathrm{cm}^{3} \mathrm{~g}^{-1}\right)^{\mathrm{b}}$ & $\mathrm{V}_{\text {micro }}\left(\mathrm{cm}^{3} \mathrm{~g}^{-1}\right)^{\mathrm{c}}$ & $\begin{array}{l}\mathrm{H}_{2} \text { capac- } \\
\text { ity }(\mathrm{wt} \%)^{\mathrm{d}}\end{array}$ & $\begin{array}{l}\text { Maximum excess } \\
\mathrm{H}_{2} \text { uptake }(\mathrm{wt} \%)^{\mathrm{e}}\end{array}$ \\
\hline PIM-1 & 338 & 341,090 & 2.7 & $864.3 \pm 1.4$ & 0.6225 & 0.2598 & 1.13 & 1.52 \\
MePIM65 & 338 & 40,995 & 1.6 & $890.8 \pm 2.4$ & 0.6404 & 0.3312 & 1.16 & - \\
MePIM150 & 423 & 60,547 & 2.2 & $921.6 \pm 0.8$ & 0.6697 & 0.3215 & 1.18 & 1.90 \\
\hline
\end{tabular}

${ }^{\text {a } D e t e r m i n e d ~ f r o m ~} \mathrm{~N}_{2}$ adsorption isotherms

${ }^{\mathrm{b}}$ Total pore volume determined by uptake at $\mathrm{p} / \mathrm{p}_{0}=0.97$ on $\mathrm{N}_{2}$ isotherms at $77 \mathrm{~K}$

${ }^{\mathrm{c}}$ Micropore volume for pores with pore size $<2 \mathrm{~nm}$

${ }^{\mathrm{d}} \mathrm{H}_{2}$ uptake measured at $0.1 \mathrm{MPa}$ and $77 \mathrm{~K}$

${ }^{\mathrm{e}}$ Maximum $\mathrm{H}_{2}$ uptake at $77 \mathrm{~K}$ and at 2.2 MPa and 5.56 MPa for PIM-1 and MePIM150, respectively

Fig. $1{ }^{1} \mathrm{H}$ NMR spectrum of PIM-1, MePIM65 and MePIM150 in $\mathrm{CDCl}_{3}$ at room temperature showing peak assignments for different protons on the molecule. The solvent peak $\left(\mathrm{CDCl}_{3}\right)$ and impurities are marked

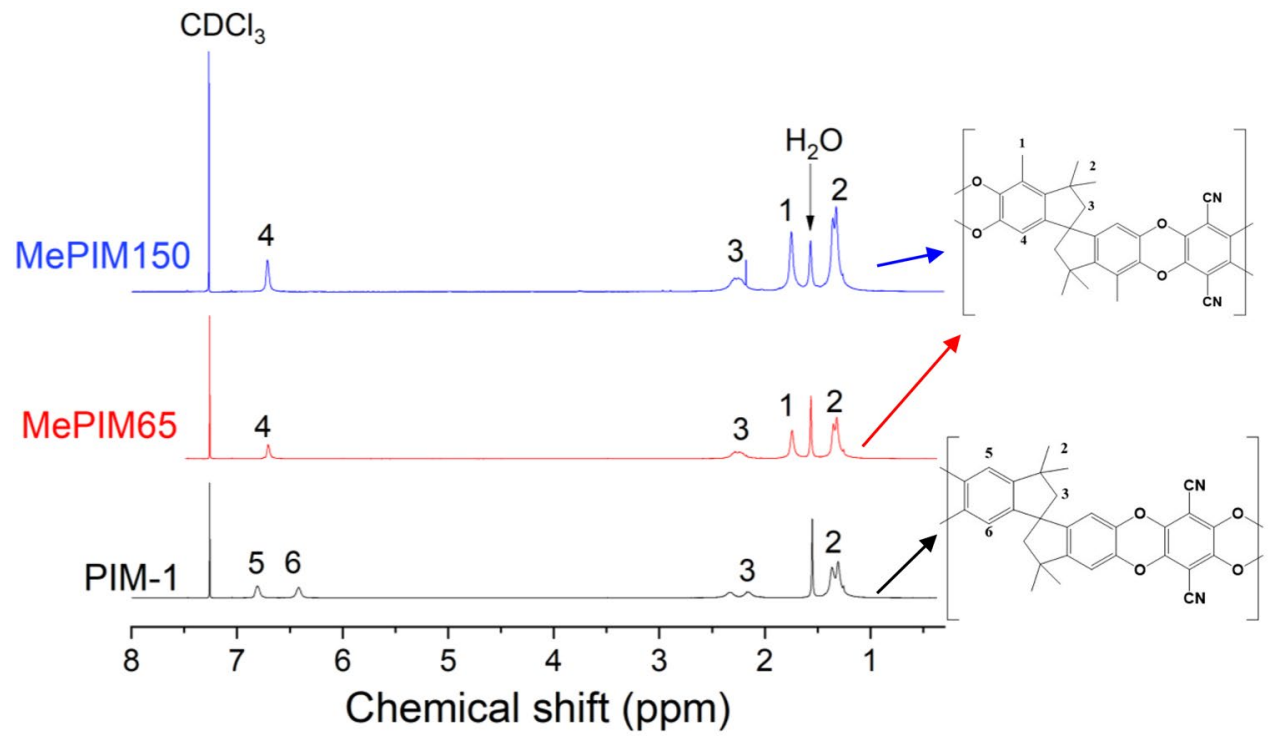

Fig. 2 Thermogravimetric analysis and of PIM-1, MePIM65 and MePIM150 in air at a heating rate of $10 \mathrm{~K} \mathrm{~min}^{-1}$ up to $1073 \mathrm{~K}$
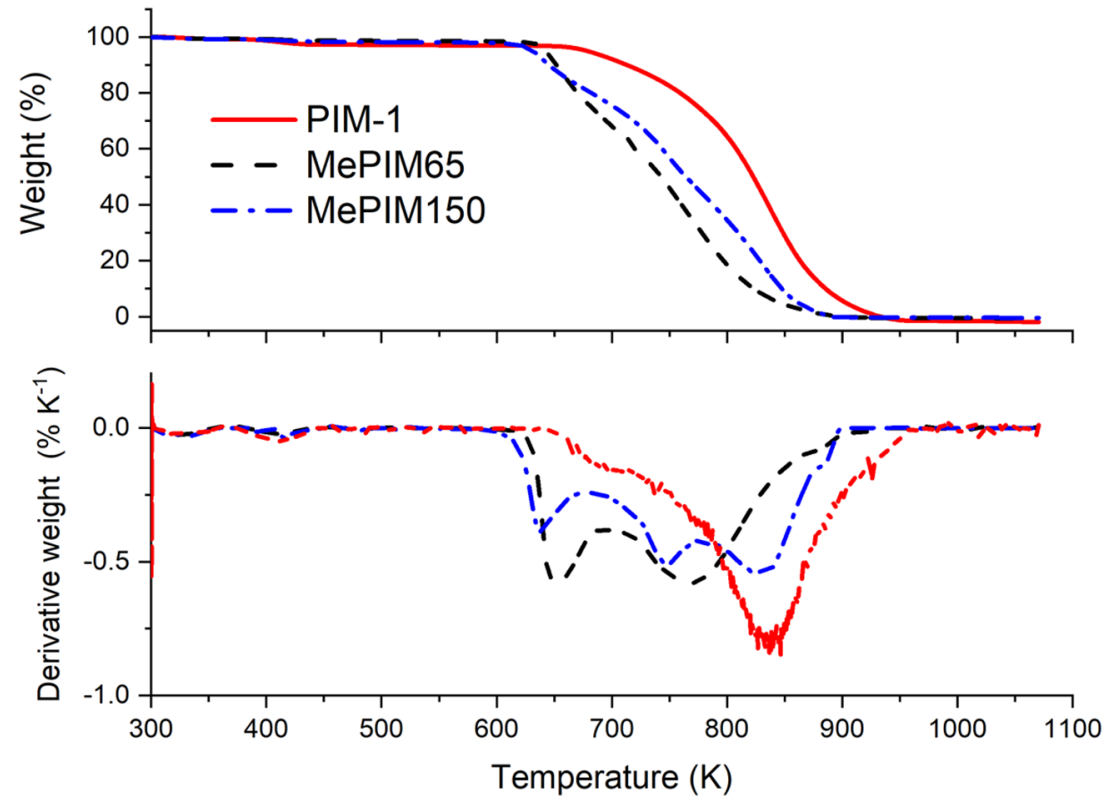

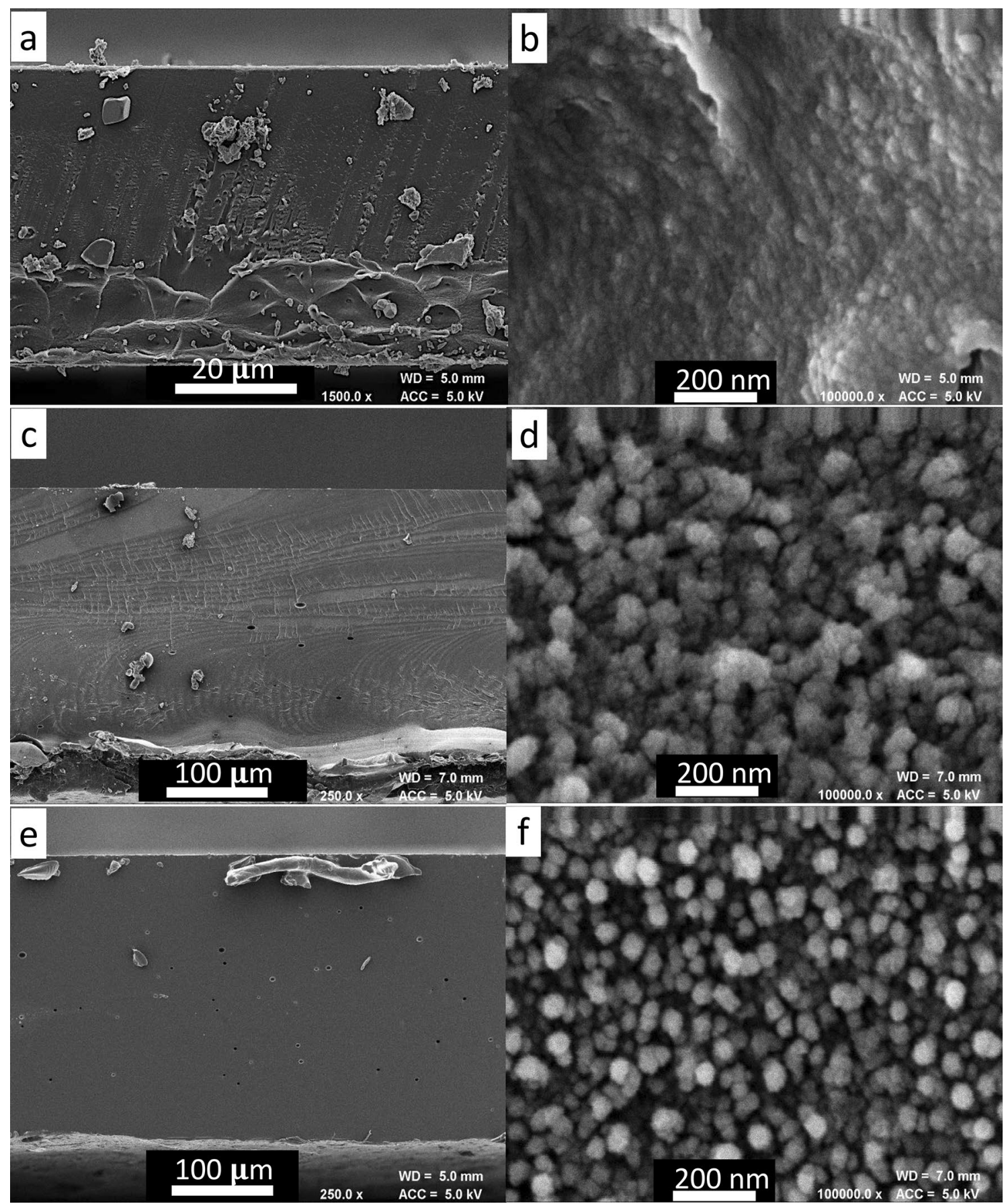

Fig. 3 Cross-section SEM images of PIM-1 (a, b), MePIM65 (c, d) and MePIM150 (e, f) membranes. Cross-sections of membrane samples were prepared by freeze fracturing after immersion in liquid nitrogen

of the MePIMs demonstrate a more granular structure, as shown in Fig. 3d, f, in comparison with the PIM-1 in Fig. 3b The more granular morphology of the MePIM films may relate to the MePIM membranes being more brittle and less thermally stable as a result of the more defective and less coherent microstructure
The surface area and total pore volume were estimated based on $\mathrm{N}_{2}$ adsorption isotherms measured at $77 \mathrm{~K}$, shown in Fig. 4. All three isotherms exhibited IUPAC Type I and Type IV behaviour in the low and medium relative pressure range. The isotherms displayed significant hysteresis, as anticipated for PIM-1 based materials due to narrow channels constricting mass transfer into mesopores. Modification 

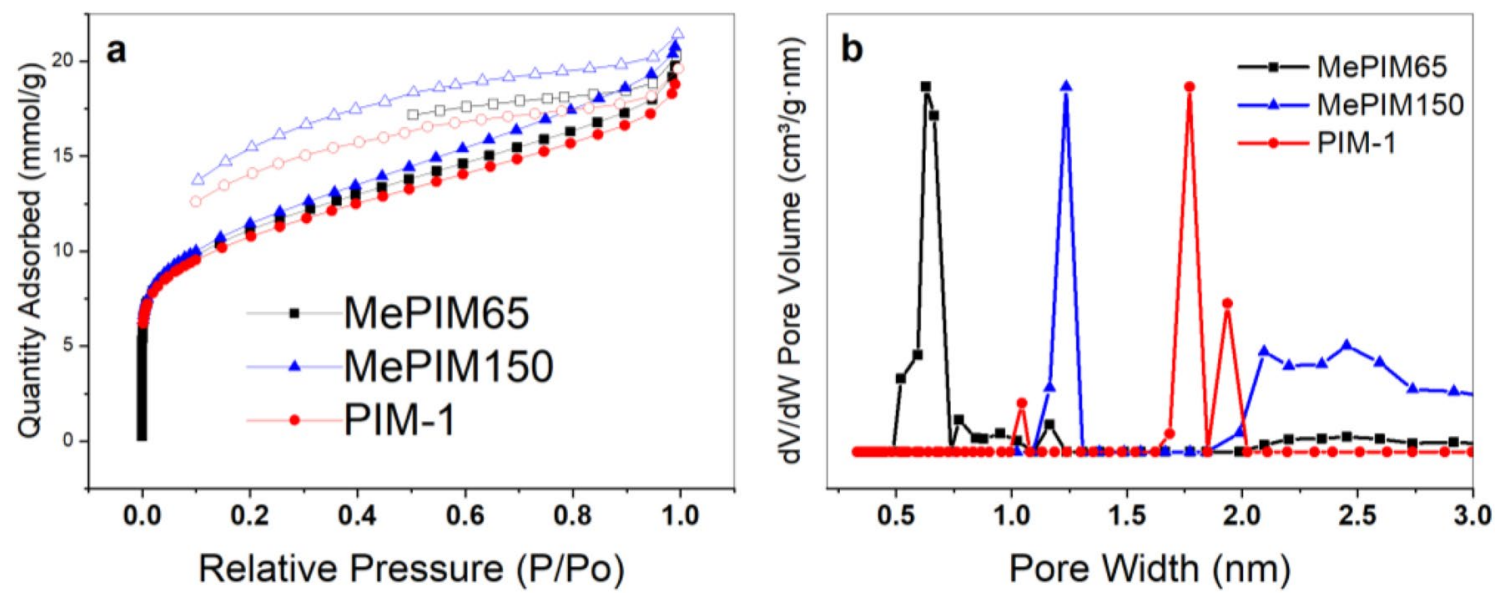

Fig. 4 a $\mathrm{N}_{2}$ isotherms for PIM-1, MePIM65 and MePIM150 carried out at $77 \mathrm{~K}$ and b micropore size distribution determined by NLDFT (slit pore model for $\mathrm{N}_{2}$ at $77 \mathrm{~K}$ )

of the PIM-1 did not affect the rate of $\mathrm{N}_{2}$ mass transfer, as indicated by the similar hysteresis loops in the $\mathrm{N}_{2}$ isotherms of MePIMs. The BET surface area determined for PIM-1 powder $\left(864 \mathrm{~m}^{2} \mathrm{~g}^{-1}\right)$ is in good agreement with prior literature values (Budd et al. 2004; Polak-Kraśna et al. 2017). BET surface areas of 891 and $921 \mathrm{~m}^{2} \mathrm{~g}^{-1}$ were determined for MePIM65 and MePIM150, respectively (Table 1). The pore size distribution (PSD) in Fig. $4 \mathrm{~b}$ demonstrates that the pores sizes of PIM-1 are distributed between 1.6 and $2.0 \mathrm{~nm}$. MePIM65 has a narrow pore size distribution centred at $0.6 \mathrm{~nm}$, as determined by NLDFT of $\mathrm{N}_{2}$ isotherm at $77 \mathrm{~K}$ and $\mathrm{CO}_{2}$ isotherm at $273 \mathrm{~K}$ (Figure S1). MePIM150 has a distribution centred at $1.2 \mathrm{~nm}$, as determined by NLDFT of $\mathrm{N}_{2}$ isotherms. It was observed that $52 \%$ and $48 \%$ of the pore volumes or surface area of MePIM65 and MePIM150 correspond to the micropore range, in contrast to $42 \%$ of microporous volume for PIM-1. Therefore, the introduction of methyl groups to the spirobisindane part of PIM-1 generates additional microporous spaces, which were anticipated to significantly benefit hydrogen storage capacity.

We investigated the hydrogen storage properties for all three materials up to $0.1 \mathrm{MPa}$ and $77 \mathrm{~K}$, and these were, as expected, directly correlated to the surface areas of the materials (Broom et al. 2016). Hydrogen uptake at $0.1 \mathrm{MPa}$ and $77 \mathrm{~K}$ of PIM-1 (1.13 wt\%), MePIM65 (1.16 wt\%) and MePIM150 (1.18 wt\%) increased with an increase of surface area, as shown in Table 1 and Fig. 5. After modification, the hydrogen uptake at $0.1 \mathrm{MPa}$ has been improved by $4 \%$ due to the increase in surface area and porosity.

High-pressure hydrogen uptake measurements were recorded on PIM-1 and on MePIM150 to investigate the effect of its superior surface area. The high pressure $\mathrm{H}_{2}$ isotherms for PIM-1 and MePIM150 at 77 K, shown in Fig. 6 and Figure S2, illustrate fully reversible adsorption processes for these materials. The maximum excess $\mathrm{H}_{2}$ uptake of 1.90

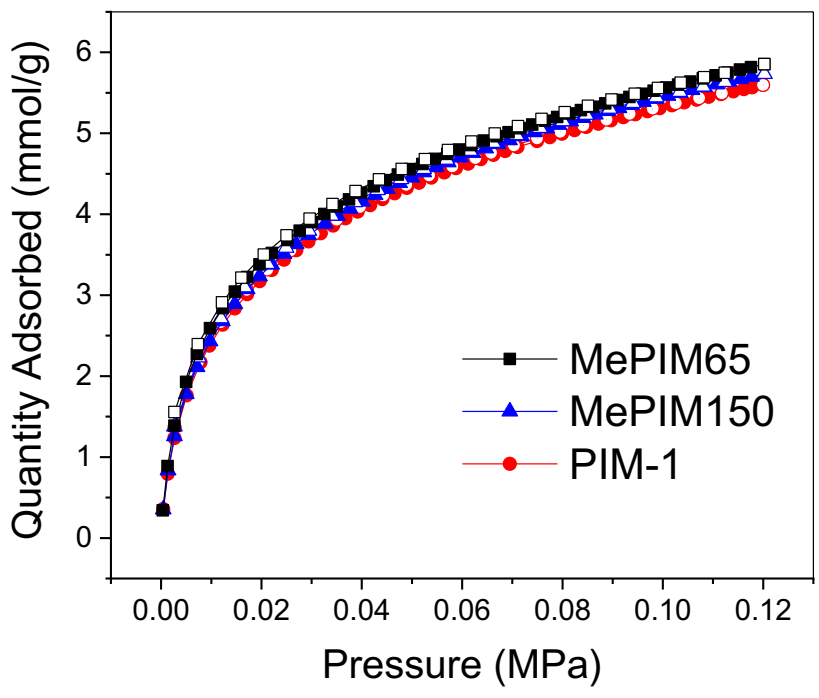

Fig. $5 \mathrm{H}_{2}$ isotherms at $77 \mathrm{~K}$ up to $0.12 \mathrm{MPa}$ for PIM-1, MePIM65 and MePIM150

wt\% was achieved at 5.56 MPa for MePIM150, which is a $25 \%$ improvement over the highest excess $\mathrm{H}_{2}$ uptake of 1.52 wt $\%$ determined for PIM-1. This increase in hydrogen capacity can be correlated to the observed increase in microporous surface in MePIM150, which results from the introduction of methyl groups to the structure of PIM-1. Using Type I isotherm fitting that was developed by our group previously (Sharpe et al. 2013), total gravimetric uptakes were calculated that provide the total amount that the materials can absorb. These are $2.9 \mathrm{wt} \%$ for PIM-1 and $3.1 \mathrm{wt} \%$ for MePIM150 at $77 \mathrm{~K}$ and $18 \mathrm{MPa}$. The introduction of methyl side groups in the contorted backbone of PIM-1 enhances the surface area and consequently improves the hydrogen storage capacity. 


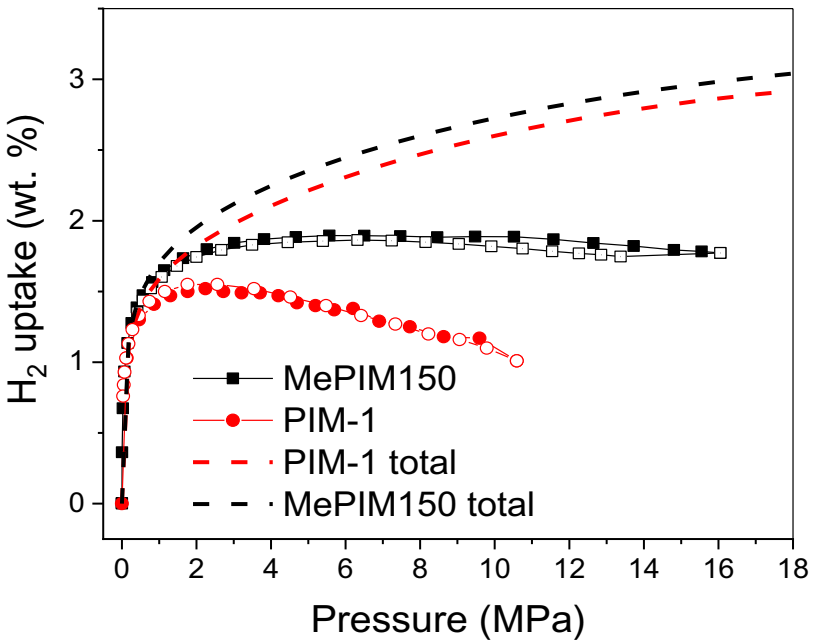

Fig. 6 High-pressure $\mathrm{H}_{2}$ excess isotherms and calculated total uptakes at $77 \mathrm{~K}$ up to $16 \mathrm{MPa}$ for PIM-1 and MePIM 150

\section{Conclusion}

This work has demonstrated the successful synthesis of a type of chemically modified PIM-1 analogues, MePIMs, by introducing methyl group to the spirobisindane part of PIM-1 and provides the first measurements of their hydrogen storage properties. We performed the synthesis under low and high temperature conditions, and detailed characterisation has demonstrated that the modification of PIM-1 resulted in enhanced surface area and porosity based on $\mathrm{N}_{2}$ isotherms measurements and analyses. The MePIMs can be cast into free standing membranes through a solvent casting method, which make these materials conveniently processable.

The introduction of methyl groups to the spirobisindane part of PIM-1 leads to the formation of additional microporous spaces, which in turn leads to a significant improvement in high-pressure hydrogen storage capacity $(1.90 \mathrm{wt} \%$ for MePIM150 vs. $1.52 \mathrm{wt} \%$ for PIM-1 at the maximum excess uptake and at $77 \mathrm{~K}$ ). The surface area and microporosity of MePIMs were 7\% and 24\% higher than those of PIM1. The MePIM150 materials show slightly higher surface areas and porosity, compared to MePIM65. Subsequently, a maximum hydrogen excess adsorption of $1.90 \mathrm{wt} \%$ was achieved at $5.56 \mathrm{MPa}$, which is a $25 \%$ higher capacity than that of PIM-1. This indicates that MePIMs are promising materials for hydrogen storage applications. Further attempts to fabricate composite MePIM membranes by introducing porous fillers for improving porosity and functionality of the MePIMs are promising next steps.
Acknowledgements This work was financially supported by the UK Engineering and Physical Sciences Research Council (EPSRC) via SUPERGEN Grants EP/K021109/1 and EP/L018365/1.

Open Access This article is licensed under a Creative Commons Attribution 4.0 International License, which permits use, sharing, adaptation, distribution and reproduction in any medium or format, as long as you give appropriate credit to the original author(s) and the source, provide a link to the Creative Commons licence, and indicate if changes were made. The images or other third party material in this article are included in the article's Creative Commons licence, unless indicated otherwise in a credit line to the material. If material is not included in the article's Creative Commons licence and your intended use is not permitted by statutory regulation or exceeds the permitted use, you will need to obtain permission directly from the copyright holder. To view a copy of this licence, visit http://creativecommons.org/licenses/by/4.0/.

\section{References}

Broom, D.P., Webb, C.J., Hurst, K.E., Parilla, P.A., Gennett, T., Brown, C.M., Zacharia, R., Tylianakis, E., Klontzas, E., Froudakis, G.E., Steriotis, T.A., Trikalitis, P.N., Anton, D.L., Hardy, B., Tamburello, D., Corgnale, C., van Hassel, B.A., Cossement, D., Chahine, R., Hirscher, M.: Outlook and challenges for hydrogen storage in nanoporous materials. Appl. Phys. A 122(3), 151 (2016)

Budd, P.M., Elabas, E.S., Ghanem, B.S., Makhseed, S., McKeown, N.B., Msayib, K.J., Tattershall, C.E., Wang, D.: Solution-processed, organophilic membrane derived from a polymer of intrinsic microporosity. Adv. Mater. 16(5), 456-459 (2004)

Budd, P.M., Msayib, K.J., Tattershall, C.E., Ghanem, B.S., Reynolds, K.J., McKeown, N.B., Fritsch, D.: Gas separation membranes from polymers of intrinsic microporosity. J. Membr. Sci. 251(1), 263-269 (2005)

Du, N., Robertson, G.P., Song, J., Pinnau, I., Guiver, M.D.: Highperformance carboxylated polymers of intrinsic microporosity (PIMs) with tunable gas transport properties. Macromolecules 42(16), 6038-6043 (2009)

Du, N., Robertson, G.P., Pinnau, I., Guiver, M.D.: Polymers of intrinsic microporosity with dinaphthyl and thianthrene segments. Macromolecules 43(20), 8580-8587 (2010)

Halder, K., Neumann, S., Bengtson, G., Khan, M.M., Filiz, V., Abetz, V.: Polymers of intrinsic microporosity postmodified by vinyl groups for membrane applications. Macromolecules 51(18), 7309-7319 (2018)

Khdhayyer, M.R., Esposito, E., Fuoco, A., Monteleone, M., Giorno, L., Jansen, J.C., Attfield, M.P., Budd, P.M.: Mixed matrix membranes based on UiO-66 MOFs in the polymer of intrinsic microporosity PIM-1. Sep. Purif. Technol. 17, 304-313 (2017)

Kim, H.J., Kim, D.-G., Lee, K., Baek, Y., Yoo, Y., Kim, Y.S., Kim, B.G., Lee, J.-C.: A carbonaceous membrane based on a polymer of intrinsic microporosity (PIM-1) for water treatment. Sci. Rep. 6, 36078 (2016)

Matthias, T., Katsumi, K., Alexander, V.N., James, P.O., Francisco, R.-R., Jean, R., Kenneth, S.W.S.: Physisorption of gases, with special reference to the evaluation of surface area and pore size distribution (IUPAC Technical Report), vol. 87(9-10), pp. 10511069 (2015)

Polak-Kraśna, K., Dawson, R., Holyfield, L.T., Bowen, C.R., Burrows, A.D., Mays, T.J.: Mechanical characterisation of polymer of intrinsic microporosity PIM-1 for hydrogen storage applications. J. Mater. Sci. 52(7), 3862-3875 (2017)

Rochat, S., Polak-Krasna, K., Tian, M., Holyfield, L.T., Mays, T.J., Bowen, C.R., Burrows, A.D.: Hydrogen storage in polymer-based 
processable microporous composites. J. Mater. Chem. A 5(35), 18752-18761 (2017). https://doi.org/10.1039/C7TA05232D

Satilmis, B., Budd, P.M.: Selective dye adsorption by chemically-modified and thermally-treated polymers of intrinsic microporosit. J. Colloid Interface Sci. 492, 81-91 (2017)

Satilmis, B., Isık, T., Demir, M.M., Uyar, T.: Amidoxime functionalized Polymers of Intrinsic Microporosity (PIM-1) electrospun ultrafine fibers for rapid removal of uranyl ions from water. Appl. Surf. Sci. 467-468, 648-657 (2019)

Sharpe, J.E., Bimbo, N., Ting, V.P., Burrows, A.D., Jiang, D., Mays, T.J.: Supercritical hydrogen adsorption in nanostructured solids with hydrogen density variation in pores. Adsorption 19(2), 643-652 (2013)

Song, Q., Cao, S., Pritchard, R.H., Ghalei, B., Al-Muhtaseb, S.A., Terentjev, E.M., Cheetham, A.K., Sivaniah, E.: Controlled thermal oxidative crosslinking of polymers of intrinsic microporosity towards tunable molecular sieve membranes. Nat. Commun. 5, 4813 (2014)

Swaidan, R., Ghanem, B.S., Litwiller, E., Pinnau, I.: Pure- and mixed-gas $\mathrm{CO} 2 / \mathrm{CH} 4$ separation properties of PIM-1 and an amidoxime-functionalized PIM-1. J. Membr. Sci. 457, 95-102 (2014)

Tian, M., Rochat, S., Polak-Kraśna, K., Holyfield, L.T., Burrows, A.D., Bowen, C.R., Mays, T.J.: Nanoporous polymer-based composites for enhanced hydrogen storage. Adsorption 25(4), 889-901 (2019)

Wang, Z.G., Liu, X., Wang, D., Jin, J.: Tröger's base-based copolymers with intrinsic microporosity for $\mathrm{CO}_{2}$ separation and effect of Tröger's base on separation performance. Polym. Chem. 5(8), 2793-2800 (2014). https://doi.org/10.1039/C3PY01608K

Xu, Y., Mao, N., Feng, S., Zhang, C., Wang, F., Chen, Y., Zeng, J., Jiang, J.-X.: Perylene-containing conjugated microporous polymers for photocatalytic hydrogen evolution. Macromol. Chem. Phys. 218(14), 1700049 (2017)

Publisher's Note Springer Nature remains neutral with regard to jurisdictional claims in published maps and institutional affiliations. 